\title{
Atomic-column scanning transmission electron microscopy analysis of misfit dislocations in GaSb/ GaAs quantum dots
}

\begin{abstract}
The structural quality of GaSb/GaAs quantum dots (QDs) has been analyzed at atomic scale by aberration-corrected high-angle annular dark-field scanning transmission electron microscopy. In particular, we have studied the misfit dislocations that appear because of the high-lattice mismatch in the heterostructure. Our results have shown the formation of Lomer dislocations not only at the interface between the GaSb QDs and the GaAs substrate, but also at the interface with the GaAs capping layer, which is not a frequent observation. The analysis of these dislocations points to the existence of chains of dislocation loops around the QDs. The dislocation core of the observed defects has been characterized, showing that they are reconstructed Lomer dislocations, which have less distortion at the dislocation core in comparison to unreconstructed ones. Strain measurements using geometric phase analysis show that these dislocations may not fully relax the strain due to the lattice mismatch in the GaSb QDs.
\end{abstract}

